\title{
Optimum QoS resource allocation algorithm for video traffic over wireless mesh networks based on IEEE 802.11s
}

\begin{abstract}
Wireless Mesh Network (WMN) is a communications network consists of radio nodes organized in a mesh topology. WMNs are gaining wide popularity due to their ability to integrate several networks in one network. It increases reliability and improved performance over conventional wireless LANs. Wi-Fi could be integrated in WMN. Several standards of IEEE 802.11 were created to support and deal with specific tasks of Wi-Fi. IEEE 802.11s has been created to support QoS in WMNs where it has been approved as a standard lately. However, it is still has some limitations in supporting QoS. In this paper we introduce our work which is built depends on previous works where all of them deal with TDMA/TDD and aims to enhance the QoS. We propose a scheduling mechanism which works in MAC layer in wireless mesh networking based on IEEE 802.11s. It gives the delayed video users the priority to serve them first while maintaining the QoS of audio and data users. This paper provides our results compared with previous works. We introduce them with a huge discussion, according to the QoS requirements.
\end{abstract}

Keyword: IEEE 802.11s; QoS; Video traffic; Wi-Fi; WMNs 